\title{
Atividade colinesterásica cerebral e comportamento de ratos após exposição perinatal ao diclorvós
}

\author{
Brain cholinesterase activity and the behavior of rats after perinatal exposure to dichlorvos
}

\author{
Jaqueline Pérola de Souza' ${ }^{1}$ Gustavo Milhomens Nogueira ${ }^{2}$ Maria Isabel Mataqueiro ${ }^{3}$ \\ Antonio de Queiroz-Neto ${ }^{4}$
}

\section{RESUMO}

\begin{abstract}
O organofosforado diclorvós impregnado em coleiras plásticas é um recurso utilizado em medicina veterinária que visa ao controle de ectoparasitas de cães e gatos. $O$ objetivo deste trabalho foi avaliar os efeitos do uso de coleiras plásticas impregnadas com diclorvós $(8,37 \%)$ em ratas Wistar durante o período de gestação e lactação, como possível fonte de alterações comportamentais e da atividade colinesterásica cerebral dos filhotes. Na desmama, não houve diferença na atividade colinesterásica cerebral entre as mães tratadas com diclorvós e o grupo controle, bem como entre os respectivos filhotes. O tratamento com diclorvós também não influenciou no comportamento geral dos animais, avaliado no campo aberto, nem no nível de ansiedade testado no labirinto em cruz elevado, ambos aos 35 dias pós-natal.
\end{abstract}

Palavras-chave: 2,2-diclorovinil dimetil fosfato, neurotoxicologia do desenvolvimento, coleira antipulga, campo aberto, labirinto em cruz elevado.

\section{ABSTRACT}

The organophosphate dichlorvos impregnated into plastic collars $(8.37 \%)$ is used in veterinary practice as an alternative for the control of ectoparasites in dogs and cats. The aim of this work was to determine the possible toxic effects of these collars in female Wistar rats during pregnancy and lactation, as a possible cause of alterations in brain cholinesterase activity and behavior of offspring. At weaning, there was no difference in brain cholinesterase activity between control and treated dams, nor between their respective offspring as well. The treatment did not affect the general behavior of the offspring, when evaluated in the open field, nor anxiety in the elevated plus-maze, both evaluated on the 35th postnatal day.

Key words: 2.2-dichlorovinyl dimethyl phosphate, developmental neurotoxicology, flea collar, open field behavior, elevated plus-maze.

\section{INTRODUÇÃO}

O composto 2,2-diclorovinil dimetil fosfato (DDVP), conhecido como diclorvós, é um organofosforado de elevada atividade praguicida, e por isso empregado como inseticida em formulações que utilizam sistemas de liberação lenta, como a impregnação a coleiras plásticas de cloreto de polivinila (PVC). Comercialmente o diclorvós tem sido produzido desde 1961 e utilizado no controle de moscas, mosquitos, baratas, pulgas, carrapatos entre outras pragas (WHO/ IPCS, 1989). Experimentos controlados com coleiras impregnadas com diclorvós demostraram a eficiência desta preparação no combate a pulgas em cães e gatos (KIBBLE, 1968; FOX et al.,1969).

Embora publicações científicas relatem sua eficácia como anti-parasitário, pouco é conhecido a respeito do potencial desta apresentação de diclorvós como fonte de incapacidades neurocomportamentais decorrentes de sua interferência no desenvolvimento cerebral. LAZARINI et al. (2004) avaliaram variáveis

\footnotetext{
${ }^{1}$ Curso de Ciências Biológicas, Faculdade de Ciências Agrárias e Veterinárias (FCAV), Universidade Estadual Paulista (Unesp), Campus de Jaboticabal, Jaboticabal, SP, Brasil.

${ }^{2}$ Faculdade de Medicina Veterinária e Zootecnia de Garça, SP, Brasil.

${ }^{3}$ FCAV, Unesp, Campus de Jaboticabal, Jaboticabal, SP, Brasil.

${ }^{4}$ Departamento de Morfologia e Fisiologia Animal, FCAV, Unesp, 14884-900, Jaboticabal, SP, Brasil. E-mail:aqueiroz@fcav.unesp.br. Autor para correspondência.
} 
comportamentais em filhotes de ratas expostas, por via oral, ao diclorvós durante o período de gestação, e observaram alterações motoras em filhotes jovens e adultos, concluindo que a exposição pré-natal a baixas doses de DDVP reduz a atividade exploratória em animais adolescentes e a atividade motora em animais adultos. O sistema nervoso central é bastante sensível a produtos químicos, especialmente nos estágios iniciais de desenvolvimento (SPYKER, 1975). Esta sensibilidade do cérebro em desenvolvimento ao insulto tóxico pode se manifestar como distúrbios sutis do comportamento que podem ser determinados por meio de técnicas de psicologia comportamental que podem registrar e quantificar fenômenos aparentemente imensuráveis como inteligência e ansiedade.

Considerando que as coleiras antipulgas são produtos que exalam vapores de diclorvós de forma prolongada e que são usadas indiscriminadamente em cadelas gestantes e/ou lactantes, existe a possibilidade de haver risco tóxico para as crias. Além disso, haja vista a grande freqüência de contato próximo entre seres humanos e seus animais de companhia, também pode haver risco toxicológico para crianças. Desta forma, objetivou-se, neste trabalho, avaliar os efeitos do uso da coleira impregnada com diclorvós em ratas Wistar durante o período de gestação e lactação como possível fonte de alterações comportamentais e da atividade colinesterásica cerebral dos filhotes.

\section{MATERIAL E MÉTODOS}

Foram utilizadas 20 ratas Wistar, sexualmente maduras, nulíparas, pesando entre 230 e 320 g, provenientes do Biotério Central da Unesp, Campus de Botucatu. Os animais foram alojados no biotério experimental do laboratório de toxicologia, Departamento de Morfologia e Fisiologia Animal da Faculdade de Ciências Agrárias e Veterinárias da Unesp Campus de Jaboticabal, em caixas plásticas medindo $16 \times 30 \times 18 \mathrm{~cm}$, mantidos em salas com temperatura controlada $\left(23 \pm 2^{\circ} \mathrm{C}\right)$ e fotoperíodo cíclico de 12 horas, com água e comida (ração para roedores ${ }^{\mathrm{a}}$ ) à vontade.

As ratas eram examinadas diariamente por meio de lavados vaginais, para verificação do ciclo estral. Eram mantidas com machos (1 macho para 2 fêmeas), e após diagnóstico de prenhez positivo, os animais do grupo tratado eram submetidos ao uso de coleiras plásticas ${ }^{\mathrm{b}}$ impregnadas com $8,37 \%$ de diclorvós padronizado em $27,5 \mathrm{~cm}$ de comprimento e peso líquido de $8,35 \mathrm{~g}$ que circundava o pescoço dos animais com duas voltas. Os animais do grupo controle eram submetidos ao uso de coleiras inertes. Foram consideradas três possíveis vias de exposição ao praguicida para as mães: a dermal, a oral (devido ao comportamento de auto-limpeza) e a inalatória; e para os filhotes a via transplacentária, oral (transferência através do leite durante a lactação), dermal e a via inalatória.

A atividade colinesterásica cerebral das ratas tratadas e controles foi avaliada ao final da lactação utilizando-se o método de ELLMAN et al. (1961) modificado conforme a seguir: o hemisfério direito do cérebro era homogenizado (aproximadamente $80 \mathrm{mg}$ de tecido por $\mathrm{ml}$ de solução de $\mathrm{NaCl} 0,9 \%$ resfriada) em tubo de ensaio com auxílio de um bastão de vidro. Estes homogenatos eram centrifugados a $12000 \mathrm{~g}$, por cinco sessões de dois minutos cada, intercaladas com resfriamento em gelo (totalizando 10 minutos de centrifugação). O sobrenadante destas amostras foi armazenado em tubos plásticos e congelados a $-5^{\circ} \mathrm{C}$ por aproximadamente cinco dias, quando eram descongelados e mantidos em banhomaria a $25^{\circ} \mathrm{C}$. Alíquotas de $20 \mu \mathrm{L}$ destes sobrenadantes eram adicionadas a cubetas contendo $3,0 \mathrm{ml}$ de tampão fosfato $0,1 \mathrm{M}-\mathrm{pH} 7,4$. Adicionava-se, então, $100 \mu \mathrm{L}$ de ácido ditiobisnitrobenzóico ${ }^{\mathrm{c}} 0,1 \mathrm{M}$ e procedia-se a leitura no espectofotômetro para a calibração, empregando-se o comprimento de onda de 412nm. Adicionava-se, a seguir, $50 \mu \mathrm{L}$ do substrato (acetiltiocolina iodada ${ }^{\mathrm{d}}$ ) à cubeta e iniciava-se o registro da absorbância a cada minuto durante cinco minutos. Calculava-se a variação da absorbância por minuto a partir da média dos valores das cinco leituras de cada amostra, e a variação da absorbância era considerada a diferença da leitura no tempo final e no tempo inicial. A atividade enzimática era expressa em moles de substrato hidrolizado por minuto por grama de tecido cerebral. A atividade colinesterásica cerebral dos filhotes, de maneira semelhante, foi quantificada aos 21 dias de idade, selecionando-se aleatoriamente um macho e uma fêmea de cada ninhada.

A avaliação comportamental foi realizada utilizando-se a arena em campo aberto ("open field"), e o labirinto em cruz elevado. Para estes testes, foram selecionados aleatoriamente 20 filhotes do grupo controle (10 machos e 10 fêmeas), e 20 filhotes do grupo tratado (10 machos e 10 fêmeas), a partir do 35 dia pósnatal.

O teste do campo aberto consistiu na mensuração das variáveis comportamentais dos indivíduos experimentais, colocados em uma arena limitada por uma parede circular segundo BROADHURST (1960). Cada animal foi colocado, individualmente, no centro da arena, e observadores cegos quanto aos tratamentos, anotaram as seguintes 
categorias comportamentais: freqüência de ambulação, levantar, auto-limpeza, defecação, micção e período de imobilidade. $\mathrm{O}$ teste foi efetuado em três sessões, com duração de cinco minutos cada sessão, e intervalo entre as sessões de aproximadamente 85 minutos.

O labirinto em cruz elevado explora o conflito de aproximação-evitação tendo sido validado como modelo animal para testes com drogas ansiolíticas por PELLOW et al. (1985). Cada animal foi colocado individualmente no centro do labirinto sempre de frente para um dos braços fechados e observado por um período de cinco minutos por um experimentador situado em outra sala provida de janelas de observação. Foram registradas as seguintes variáveis e categorias: número de entrada nos braços fechados e nos braços abertos, tempo de permanência nos braços fechados e nos braços abertos.

$\mathrm{O}$ teste $\mathrm{t}$ de Student foi empregado para comparar as médias da atividade colinesterásica materna. $\mathrm{Na}$ análise dos dados referentes à atividade colinesterásica dos filhotes e à medida da ansiedade no labirinto em cruz elevado, empregou-se um esquema fatorial $2 \times 2$ (dois grupos por sexo), em um delineamento inteiramente casualizado (DIC). Os dados da atividade geral em campo aberto foram analisados utilizando-se um esquema em parcelas subdivididas no tempo. Assim as parcelas foram formadas pelo fatorial "grupo x sexo" (2x2) e as subparcelas pelo tempo (três sessões), em DIC. Para a comparação das médias das características analisadas referentes aos tratamentos e sessões foi utilizado o teste de Tukey a $5 \%$ de probabilidade.

\section{RESULTADOS E DISCUSSÃO}

A atividade colinesterásica cerebral nas fêmeas expostas à coleira impregnada de diclorvós, durante o período de gestação e lactação, não apresentou diferença estatisticamente significante $(0,54 \pm 0,3 \mu \mathrm{mol}$ de substrato hidrolizado $/ \mathrm{min} / \mathrm{g}$ tecido, $\mathrm{n}=10$ ) quando comparada à atividade colinesterásica cerebral média das mães pertencentes ao grupo controle $(0,60 \pm 0,3 \mu \mathrm{mol}$ de substrato hidrolisado/min/ g tecido, $\mathrm{n}=10$ ) quando avaliada aos 22 dias pós-natal. Da mesma forma, aos 21 dias pós-natal, a atividade colinesterásica cerebral média dos filhotes das ratas tratadas, também não diferiu dos filhotes das mães controles. Não houve diferença significativa na resposta da atividade colinesterásica cerebral entre os sexos (Figura 1).

Com relação aos experimentos no campo aberto (Tabela 1), observou-se que, nas três sessões, não houve diferença significativa, dentro das sessões, entre os animais tratados e controles no que se refere às freqüências de ambulação, tempo de imobilidade, freqüência de levantar, freqüência de defecação, freqüência de micção e nem para a freqüência de autolimpeza.

Houve diferença significativa entre os sexos na freqüência de levantar. Esta diferença se manifestou na primeira e na terceira sessão (dados não mostrados). Observou-se que as fêmeas exploraram mais verticalmente do que os machos, nos momentos acima referidos. Entretanto, não foi observada interação sexo $\mathrm{x}$ tratamento. No que se refere às freqüências de ambulação, tempo de imobilidade e freqüências de levantar, defecação e micção, observaram-se diferenças significativas entre as sessões, fato não observado apenas para a freqüência de auto-limpeza que não variou entre as sessões. Vale lembrar que todas as diferenças observadas entre as sessões não apresentam relação com os tratamentos.

$\mathrm{Na}$ avaliação realizada com o labirinto em cruz elevado, cuja finalidade foi medir o nível de ansiedade dos animais tratados ou não, observou-se que a exposição das ratas ao praguicida, não alterou a performance de seus filhotes. Verificou-se que não houve interação entre os fatores tratamento e sexo, bem como não houve diferença entre os sexos para ambos os parâmetros: porcentagem de entrada e porcentagem de tempo despendido nos braços abertos (Figura 2).

No presente estudo, verificou-se que as ratas expostas às coleiras impregnadas de diclorvós durante 42 dias de tratamento não apresentaram redução estatisticamente significante na atividade colinesterásica cerebral em relação ao grupo controle.

A atividade colinesterásica cerebral dos filhotes, medida aos 21 dias pós-natal, não foi alterada com o tratamento das mães com o diclorvós. A medida da atividade colinesterásica cerebral foi realizada quando as coleiras estavam sendo usadas pelas mães já há 42 dias, sendo possível que os níveis de exposição aos vapores do praguicida tenham sido baixos ao longo de todo o período pós natal e, portanto insuficientes para induzir inibição da atividade colinesterásica cerebral no momento avaliado. Uma outra alternativa a ser considerada é uma redução dos níveis da atividade colinesterásica na fase inicial da exposição ao diclorvós, acompanhada de uma recuperação ao longo do tempo. WALKER \& STEVENSON (1968) ao testarem a segurança das coleiras plásticas contendo diclorvós em cães, monitoraram semanalmente a atividade colinesterásica plasmática e eritrocítica destes animais antes e após a colocação das coleiras, por até 19 semanas. Houve uma redução da atividade colinesterásica plasmática e eritrocítica logo na primeira 


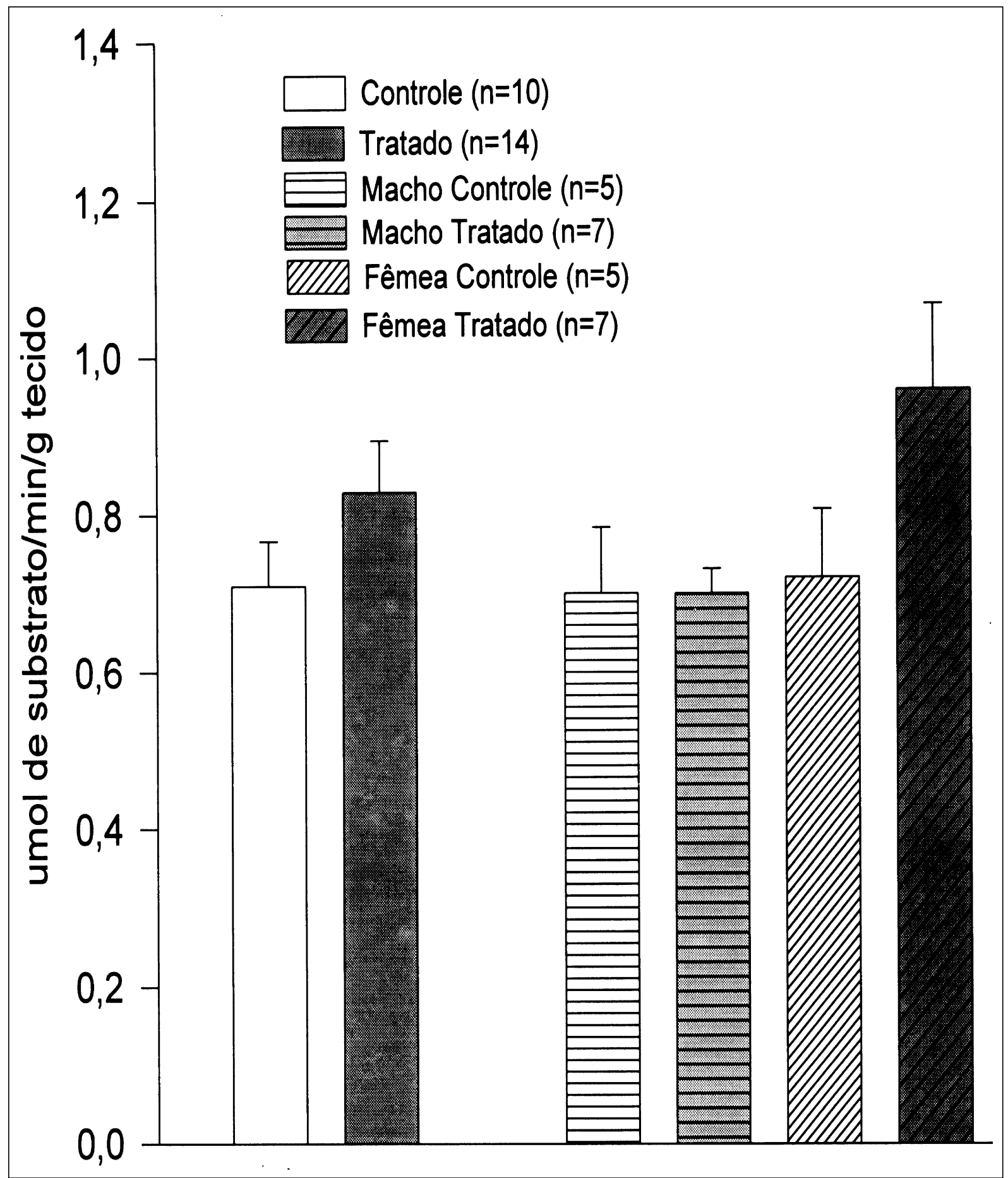

Figura 1 - Atividade colinesterásica cerebral dos filhotes de ratas tratadas ou não com diclorvós $(8,37 \%)$ impregnado em coleiras plásticas aos 21 pós-natal. As barras verticais indicam o erro padrão da média. 
Tabela 1 - Atividade (média \pm erro padrão da média) dos filhotes de ratas tratadas ou não com diclorvós impregnado em coleiras plásticas, durante sessão de cinco minutos, em uma arena de campo aberto, aos 35 dias de idade.

\begin{tabular}{|c|c|c|c|c|c|c|}
\hline \multirow{3}{*}{ Variáveis } & \multicolumn{6}{|c|}{ Sessões } \\
\hline & \multicolumn{2}{|c|}{1} & \multicolumn{2}{|c|}{2} & \multicolumn{2}{|c|}{3} \\
\hline & Controle & Tratado & Controle & Tratado & Controle & Tratado \\
\hline $\begin{array}{l}\text { Freqüência de } \\
\text { ambulação }\end{array}$ & $41,9 \pm 8,9^{\mathrm{a}}$ & $54,2 \pm 8,7^{\mathrm{a}}$ & $17,4 \pm 5,3^{b}$ & $18,8 \pm 5,0^{b}$ & $11,6 \pm 3,8^{b}$ & $11,7 \pm 3,5^{b}$ \\
\hline $\begin{array}{l}\text { Freqüência de } \\
\text { levantar }\end{array}$ & $6,2 \pm 1,3^{\mathrm{a}}$ & $7,5 \pm 1,3^{\mathrm{a}}$ & $2,1 \pm 1,0^{\mathrm{b}}$ & $1,6 \pm 0,5^{\mathrm{b}}$ & $1,9 \pm 0,7^{b}$ & $1,7 \pm 0,1^{\mathrm{b}}$ \\
\hline $\begin{array}{l}\text { Freqüência de } \\
\text { auto-limpeza }\end{array}$ & $9,4 \pm 3,5^{\mathrm{a}}$ & $12,0 \pm 3,7^{\mathrm{a}}$ & $11,6 \pm 5,1^{\mathrm{a}}$ & $13,7 \pm 4,3^{\mathrm{a}}$ & $19,2 \pm 5,9^{\mathrm{a}}$ & $12,2 \pm 2,7^{\mathrm{a}}$ \\
\hline $\begin{array}{l}\text { Período de } \\
\text { imobilidade }\end{array}$ & $205,5 \pm 25,0^{\mathrm{ab}}$ & $172,7 \pm 21,8^{\mathrm{a}}$ & $242,2 \pm 12,2^{\mathrm{bc}}$ & $244,9 \pm 13,6^{\mathrm{bc}}$ & $263,0 \pm 9,6^{c}$ & $262,9 \pm 7,9^{\mathrm{c}}$ \\
\hline $\begin{array}{l}\text { Freqüência de } \\
\text { defecação }\end{array}$ & $2,8 \pm 0,5^{\mathrm{a}}$ & $2,6 \pm 0,7^{\mathrm{a}}$ & $1,8 \pm 0,6^{\mathrm{ab}}$ & $0,8 \pm 0,4^{\mathrm{b}}$ & $1,7 \pm 0,4^{\mathrm{ab}}$ & $1,1 \pm 0,5^{\mathrm{b}}$ \\
\hline $\begin{array}{l}\text { Freqüência de } \\
\text { micção }\end{array}$ & $0,8 \pm 0,1^{\mathrm{a}}$ & $0,6 \pm 0,2^{\mathrm{ab}}$ & $0,4 \pm 0,1^{\mathrm{ab}}$ & $0,2 \pm 0,1^{b}$ & $0,6 \pm 0,2^{\mathrm{ab}}$ & $0,4 \pm 0,2^{\mathrm{b}}$ \\
\hline
\end{tabular}

Médias, na mesma linha, seguidas de letras iguais não diferem estatisticamente entre si $(\mathrm{P}>0,05)$

semana, seguida de uma recuperação gradativa ao longo das 19 semanas seguintes.

A exposição inalatória ao diclorvós a partir de coleiras plásticas apresenta algumas particularidades em relação à regularidade de liberação da substância ativa. Embora as coleiras emitam vapores de diclorvós continuamente por um período de até três meses, a taxa de liberação de diclorvós é desigual ao longo deste tempo. $\mathrm{O}$ diclorvós migra continuamente para a superfície das coleiras, mesmo quando estas encontram-se acondicionadas na embalagem antes do uso (WITCHEY-LAKSHAMANAN, 1999). Assim, uma vez que o invólucro é rompido e a coleira é aplicada no animal, uma grande quantidade de diclorvós é liberada no início, podendo, com mais probabilidade, causar problemas de toxicidade para o animal neste período. Entretanto, com o passar do tempo a concentração de diclorvós diminui dentro da resina, levando a uma redução gradual da taxa de liberação.

Avaliando-se a atividade locomotora dos filhotes no labirinto em campo aberto, observou-se que ocorreu uma diminuição desta atividade após a primeira sessão do teste, porém este resultado não esteve relacionado com o tratamento. Da mesma forma, e consistentemente com este resultado, verificou-se um aumento do período de imobilização conforme se sucederam as sessões. Esses resultados são concordantes com os de MATAQUEIRO et al. (2004) que observaram que, na primeira vez, que os animais são colocados na arena, o seu comportamento pode ser classificado de "atividade exploratória", quando os animais se movimentam bastante explorando o novo ambiente. Nas sessões subseqüentes, já estando familiarizados com a arena, o comportamento exploratório cessa e a atividade locomotora fica reduzida. A redução da freqüência de levantar-se observada nas sessões subsequentes à primeira, independente do tratamento, também pode ser explicada como uma diminuição do interesse dos animais pelo ambiente agora conhecido. Esses resultados sugerem que a coleira de diclorvós colocada nas ratas no início da gestação não afeta a atividade exploratória e locomotora de seus filhotes aos 35 dias de idade.

O fato das fêmeas apresentarem maior freqüência de levantar-se em relação aos machos na primeira e terceira sessão, independentemente do tratamento, são concordantes com as observações de ARCHER (1975) que evidenciaram um comportamento mais exagerado das fêmeas nas freqüências de levantar e locomover, quando comparadas aos machos. As freqüências de defecação e micção não foram afetadas pelo uso da coleira sendo que a diminuição dessas freqüências em função das sessões poderia ser explicada pelo esvaziamento intestinal fisiológico ou pela adaptação emocional dos animais à situação estressante a que foram submetidos. Entretanto, essa hipótese é menos provável uma vez que a freqüência de auto-limpeza, que é uma outra maneira de se avaliar a emocionalidade, permaneceu igual ao longo das sessões.

Para avaliação da ansiedade dos filhotes das ratas que foram submetidas ao tratamento, foi empregado, também, o teste do labirinto em cruz 


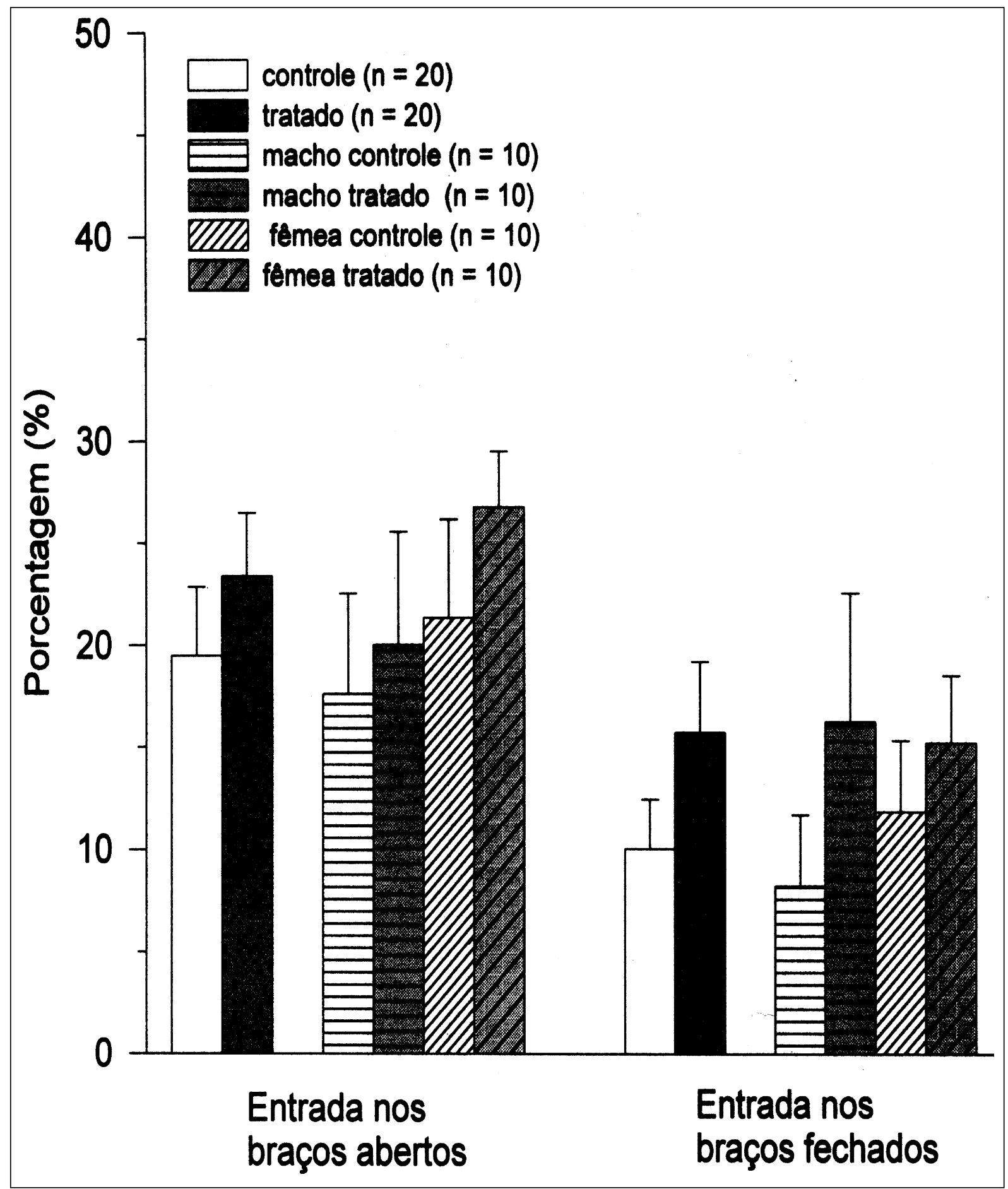

Figura 2 - Porcentagens de entrada nos braços abertos, e do tempo despendido nos braços abertos, avaliados no labirinto em cruz elevado dos filhotes de ratas tratadas ou não com diclorvós $(8,37 \%)$ impregnado em coleiras plásticas aos 35 dias pós-natal. As barras verticais indicam o erro padrão da média. 
elevado, aos 35 dias após o nascimento. Os resultados demonstraram que o desempenho dos ratos não foi modificado. É provável que o cérebro processe diversos mecanismos no controle da ansiedade, e que estes poderiam ser diferentemente afetados pelo diclorvós.

Estudos em ratos recém nascidos mostraram forte correlação temporal e regional entre a atividade da acetilcolinesterase e o desenvolvimento de conexões talamocorticais, que a princípio, não se trata de um sistema colinérgico, e nem colinoceptivo (ROBERTSON,1987). Segundo esse autor, as áreas do córtex cerebral que manifestaram atividade acetilcolinesterásica foram o córtex somatosensorial, o córtex visual primário e o córtex auditivo primário, sugerindo que a acetilcolinesterase possui um papel fundamental no desenvolvimento destas áreas. Ainda segundo esse autor, este padrão transitório da atividade da acetilcolinesterase tem início durante a primeira semana pós-natal, alcança pico de intensidade durante a segunda semana pós-natal e declina ao nível dos adultos ao final da terceira semana pósnatal. Isto nos leva a supor que bloqueios na função da acetilcolinesterase, particularmente no período inicial do desenvolvimento pós-natal, possam interferir de algum modo em funções sensoriais como a visão e a audição. Estas funções sensoriais são especialmente importantes no paradigma do campo aberto e no labirinto em cruz elevado, uma vez que os animais precisam ver e ouvir, entre outros processos de percepção, para incorporar o máximo de informações durante a exploração do novo ambiente.

O fato de não se ter observado alterações importantes que possam ser imputadas ao uso da coleira impregnada com diclorvós pode estar relacionado ao período crítico de exposição ao agente. Evidências têm sugerido que o período de maior sensibilidade do sistema nervoso central em desenvolvimento às ações tóxicas dos compostos organofosforados é sobretudo pós-natal, sendo que, nesta fase do experimento a coleira já havia perdido muito da sua substância ativa. Foi proposto que a acetilcolinesterase, enzima alvo do diclorvós, participa ativamente do desenvolvimento neuronal tanto de sistemas colinérgicos como de sistemas nãocolinérgicos, e estas ações ocorrem após o nascimento (LAUDER \& SCHAMBRA, 1999).

Em ratos, a ontogenia dos neurônios colinérgicos centrais tem início ainda na fase embriônica, sendo primeiramente evidenciada no cordão espinal e tronco encefálico entre os dias 11 e 14 pré-natais (LAUDER \& SCHAMBRA, 1999). Porém, eventos importantes deste sistema, tais como as projeções colinérgicas a partir do antecérebro basal para o neocórtex e o hipocampo, ocorrem nas duas primeiras semanas pós-natal(BERGER-SWEEMEY, 1998).

\section{CONCLUSÃO}

O uso da coleira plástica impregnada com diclorvós, na forma com foi avaliada (colocada no início da gestação) não causou alterações nos parâmetros analisados, porém considerando-se as características de volatilidade do produto e dos períodos críticos de exposição faz-se necessário novos experimentos contemplando diferentes momentos de exposição ao praguicida.

\section{FONTES DE AQUISIÇÃO}

a- Rações para animais de laboratório - Purina Labina, Agribrands do Brasil Ltda, Paulínia, SP.

b- Coleira antipulgas para cães - DDVP Purina Tratto, Nestlê Purina Pet Care, unidade. Ribeirão Preto, SP, n( da partida 009/ 2, data de fabricação: jan/2002, validade: jan/2005

c- Ácido ditiobisnitrobenzóico (DTNB): Sigma( D 8130; Sigma Aldrich Quimica Ltda, São Paulo, SP.

d- Acetiltiocolina iodada: Sigma( A 5751; Sigma Aldrich Quimica Ltda, São Paulo, SP.

O presente trabalho está de acordo com os Princípios Éticos na Experimentação Animal, adotado pelo Colégio Brasileiro de Experimentação (COBEA) e foi aprovado pela Comissão de Ética e Bem Estar Animal (CEBEA), da Faculdade de Ciências Agrárias e Veterinárias, da Unesp, Campus de Jaboticabal.

\section{REFERÊNCIAS}

ARCHER, J. Rodent sex differences in emotional and related behaviour. Behav Biol, v.14, p.451-459, 1975.

BERGER-SWEENEY, J. The effects of neonatal basal forebrain lesions on cognition: towards understanding the developmental role of the cholinergic basal forebrain. Int J Dev Neurosc, v.16, n.7/8, p.603-612, 1998

BROADHURST, P.L. Experiments in psychogenetics. In: EISENK, H.J. Experiments in Personality. London: Routledge and Kegan Paul, 1960. p.31-71.

ELLMAN G.L. et al. A new and rapid colorimetric determination of acetylcholinesterase activity. Biochem Pharmacol, v.7, p.88-95, 1961.

FOX, I. et al. Cat flea control through use of diclorvosimpregnated with collars. J Am Vet Med Assoc, v.155, n.10, p.1621-1623, 1969.

KIBBLE, R.M. Effectiveness of dichlorvos-impregnated collars in controlling fleas on dog. Aust Vet J, v.44, p.456-458, 1968.

Ciência Rural, v.36, n.2, mar-abr, 2006. 
LAUDER, J.M.; SCHAMBRA, U.B. Morphogenic roles of acetylcholine. Environ Health Perspect, v.107, p.65-69, suppl.1, p.65-69, 1999.

LAZARINI et al. Prenatal exposure to dichlorvos: physical and behavioral effects on rat offspring. Neurotoxicol Teratol, v.26, p.607-614, 2004.

MATAQUEIRO, M.I. et al. Comparative study of the sedative and antinociceptive effects of levomepromazine, azaperone ando midazolan in laboratory animals. Arq Bras Med Vet Zootec, v.56, n.3, p.340-345, 2004.

PELLOW, S. et al. Validation of open-closed arm entries in an elevated pluz-maze as a measure of anxiety in the rat. $\mathbf{J}$ Neurosci Meth, v.14, p.149-167, 1985.
ROBERTSON, R.T. A morphogenic role for transiently expressed acetylcolinesterase in developins thalamocortical systems. Neurosci Lett, v.75, p.259-264, 1987.

SPYKER, J.M. Assessing the impact of low level chemicals on development: behavioral and latent effects. Fed Proc, v.34, n.9, p.1835-1844, 1975 .

WALKER, A.I.T.; STEVENSON, D.E. Studies on safety of plastic dog collars containg dichlorvos. Vet Rec, v.83, p.538$541,1968$.

WITCHEY-LAKSHMANAN L.C. Long-acting control of ectoparasites: a review of collar technologies for companion animals. Adv Drug Deliver Rev, v.38, p.113-122, 1999.

WHO/IPCS. Enviromental health criteria 79: Dichlorvos. Geneva: World Health Organization, 1989. p.73. 